\title{
Error analysis of backscatter from discrete dipole approximation for different ice particle shapes
}

\author{
Chun-Lei Liu *, Anthony J. Illingworth \\ JCMM, Department of Meteorology, University of Reading, Reading, RG6 6BB, UK \\ Received 10 July 1996; accepted 14 February 1997
}

\begin{abstract}
Ice sphere backscatter has been calculated using both Mie theory and the discrete dipole approximation (DDA) at a wavelength of $3.2 \mathrm{~mm}(94 \mathrm{GHz})$. The electric dipole, magnetic dipole and electric quadrupole contributions to spherical particle backscatter have been analyzed. The results show that there is a resonance area around particle size parameter of 1.5 , where the calculated backscatter errors are very large due to the neglect of the magnetic dipole, and this is confirmed by applying Mie theory to $8.66 \mathrm{~mm}(35 \mathrm{GHz})$ and $3.21 \mathrm{~cm}$ (X-band) wavelengths. Based on the backscatter calculation using a cube and a hexagon column randomly oriented in space, it was found that the backscatter error from the inaccurate representation of the particle surface shape is much smaller than that from the neglect of the magnetic dipole, and the resonance occurs at different particle sizes depending upon the exact particle shapes. At a wavelength of 3.2 $\mathrm{mm}$, the particle shape has little effect on backscatter when volume-equivalent spherical particle radius $r_{\mathrm{v}}<500 \mu \mathrm{m}$, and Rayleigh backscatter can be used as a reasonable approximation for $r_{\mathrm{v}}<300 \mu \mathrm{m}$. (C) 1997 Elsevier Science B.V.
\end{abstract}

\section{Introduction}

Cirrus clouds play an important role in climate variation (Liou, 1986). The sign of the cirrus cloud feedback to climatic variation is sensitive to the effective ice particle radius and the ice water content (IWC) (Stephens et al., 1990). Using remote sensing, the effective ice particle radius can be determined by the radar/lidar backscatter ratio (Intrieri et al., 1993), and IWC can be estimated using the effective ice particle radius and radar reflectivity $Z$ (Atlas et al., 1995; Brown et al., 1995) or by $Z$ and cloud temperature $T$ (Liu et al., 1997), but all these procedures depend upon the accurate

\footnotetext{
* Corresponding author.
} 
determination of radar reflectivity $Z$, which is not only sensitive to the particle density distributions (Liu and Illingworth, 1997), but is also expected to be affected by different ice particle shapes in cirrus clouds (Evans and Stephens, 1995).

In previous studies of IWC retrieval from radar reflectivity $Z$, most papers assume ice particles are spherical (Atlas et al., 1995; Brown et al., 1995). This is not realistic in nature, because the ice crystals in cirrus clouds have many different shapes, such as column, plate, bullet, bullet rosette, and other various complicated structures (Magono and Lee, 1966; Heymsfield and Platt, 1984), and their optical properties can not be calculated exactly using the Maxwell electromagnetic equations due to their complicated structures.

For non-spherical particle scattering at millimetre wavelength, calculations have been carried out using the discrete dipole approximation (DDA) which was first described by Purcell and Pennypacker (1973). The particle is regarded as consisting of a large number of small cubic electric dipoles, and the scattering field is the summation of contributions from these electric dipoles. Recently, the DDA method has been widely modified and employed by many investigators, such as Singham and Salzman (1986), Singham and Bohren (1987), Draine (1988), Goedecke and O'Brien (1988), Dungey and Bohren (1993) and Flatau et al. (1993). A review of this method was given by Draine and Flatau (1994). More recently, Schneider and Stephens (1995) applied the DDA method to test the limit of the Rayleigh scattering approximation and the validity of spheroidal models to model semirealistic cirrus particles and showed that spheroids are reasonable models of cirrus ice particles at wavelengths of 3 and $8 \mathrm{~mm}$. Furthermore, they pointed out that with careful consideration of optical size it is possible to exploit the Rayleigh approximation for spheroids under many circumstances. Evans and Stephens (1995) applied the DDA method to several ice particle shapes to calculate particle extinction and scattering. Their results indicated that the DDA can calculate the scattering properties of different particle shapes with adequate accuracy. They also found that the particle shape has a significant effect on the ice particle scattering within micro-wavelength regions, and the crystal shape has the dominant effect on the polarization of the scattering.

Only electric dipoles are considered in the DDA method, the magnetic dipoles and higher electric and magnetic multipoles are neglected, so the calculated backscatter from DDA code is only an approximation. In order to give confidence in applying the DDA to ice particle backscatter calculations, it is necessary to understand this error and its dependence on the particle size. Okamoto et al. (1995) have carried out the error analysis using the DDA method for hexagonal columns at $94 \mathrm{GHz}$, and suggested that the backscatter errors due to neglecting higher orders of multipole terms in the DDA calculations are much smaller than the effect of the discretization of the particle surface by the cubic array of dipoles. Following their procedures, this has been further investigated in this report by applying the DDA method to spherical, cubic and hexagonal column ice particles, in order to understand the DDA error source in its application to ice particle backscatter calculations at millimetre radar wavelengths, and we found that their conclusions are only true for small particles. When the particle size increases, there will be a resonance region where the backscatter error due to neglecting higher orders of multipole terms in the DDA calculations is much larger than that due to the discretization of the particle surface. 


\section{Theory}

Following Okamoto et al. (1995), we compare the backscatter results from the DDA and Mie theory (Mie, 1908) using spherical particles, in order to see the effect of neglecting higher order multipole terms, such as the magnetic dipole moment, electric quadrupole and so on. Based on the Mie theory (see Bohren and Huffman, 1983), backscatter intensity $i_{\mathrm{b}}$ for a sphere is

$$
i_{\mathrm{b}(\mathrm{Mie})}=\left|S_{\mathrm{b}}\right|^{2}
$$

where $S_{\mathrm{b}}$ is the backscatter amplitude, and can be expressed in terms of $a_{n}$ and $b_{n}$ by

$$
S_{\mathrm{b}}=\frac{1}{2} \sum_{n=1}^{\infty}(2 n+1)(-1)^{n}\left(a_{n}-b_{n}\right)
$$

where

$$
\begin{aligned}
& a_{n}=\frac{m \psi_{n}\left(m X_{\mathrm{d}}\right) \psi_{n}^{\prime}\left(X_{\mathrm{d}}\right)-\psi_{n}\left(X_{\mathrm{d}}\right) \psi_{n}^{\prime}\left(m X_{\mathrm{d}}\right)}{m \psi_{n}\left(m X_{\mathrm{d}}\right) \xi_{n}^{\prime}\left(X_{\mathrm{d}}\right)-\xi_{n}\left(X_{\mathrm{d}}\right) \psi_{n}^{\prime}\left(m X_{\mathrm{d}}\right)} \\
& b_{n}=\frac{\psi_{n}\left(m X_{\mathrm{d}}\right) \psi_{n}^{\prime}\left(X_{\mathrm{d}}\right)-m \psi_{n}\left(X_{\mathrm{d}}\right) \psi_{n}^{\prime}\left(m X_{\mathrm{d}}\right)}{\psi_{n}\left(m X_{\mathrm{d}}\right) \xi_{n}^{\prime}\left(X_{\mathrm{d}}\right)-m \xi_{n}\left(X_{\mathrm{d}}\right) \psi_{n}^{\prime}\left(m X_{\mathrm{d}}\right)}
\end{aligned}
$$

where $\Psi_{n}$ and $\xi_{n}$ are Riccati-Bessel functions. $m$ is the particle refractive index depending upon the incident wavelength $(\lambda) . X_{d}$ is the particle size parameter $(=2 \pi r / \lambda$, $r$ is the particle radius). $a_{1}, b_{1}$ and $a_{2}$ correspond to electric dipole, magnetic dipole and electric quadrupole moment, respectively. The fractional error $(e)$ due to neglect of other multipole terms can be defined by

$$
e=\frac{i}{i_{\mathrm{b}(\mathrm{Mie})}}-1
$$

where $i$ can be the backscatter intensity from electric dipole $i\left(a_{1}\right)$, magnetic dipole $i\left(b_{1}\right)$, or the electric quadrupole $i\left(a_{2}\right)$ in Mie theory. When this fractional error is applied to other particle shapes, $i$ will be the backscatter intensity from DDA, and $i_{\mathrm{b}(\mathrm{Mic})}$ will be replaced by the assumed true backscatter intensity from DDA.

The DDA method requires that the cubic cell dimension $d$ should satisfy the following criterion (Goedecke and O'Brien, 1988; Okamoto et al., 1995):

$$
d \leq \frac{0.5 \lambda}{2 \pi|m|}
$$

and this is easily satisfied in millimetre wavelength region.

The wavelengths and associated solid ice particle refractive indices used in our calculations are listed in Table 1. The refractive index of ice is taken from Liebe et al. (1989). Okamoto et al. (1995) used the refractive index from Ray (1972), which has different values for the imaginary component. The results in this paper are not sensitive 
Table 1

Radar wavelength and associated solid ice refractive indices

\begin{tabular}{lll}
\hline Radar & Wavelength & Index of refraction \\
\hline $94 \mathrm{GHz}$ & $3.2 \mathrm{~mm}$ & $1.784-\left(2.28 \times 10^{-3}\right) i$ \\
$K_{\mathrm{a}}$-band & $8.66 \mathrm{~mm}$ & $1.784-\left(8.44 \times 10^{-4}\right) i$ \\
X-band & $3.21 \mathrm{~cm}$ & $1.784-\left(2.39 \times 10^{-4}\right) i$ \\
\hline
\end{tabular}

to this difference. Unless otherwise stated, the ice particle will be solid throughout our calculations.

\section{Results}

The best way to investigate errors in the DDA code is to compare DDA results with known results, such as results from Mie theory using spherical ice particles. Fig. 1 is the backscatter fractional error of spherical particles under various calculation conditions. The spherical particle radius varies from 1 to $2000 \mu \mathrm{m}$, which represents the particle size range in natural cirrus clouds, and the wavelength used here is $3.2 \mathrm{~mm}(94 \mathrm{GHz})$. The true value $i_{\mathrm{b}(\mathrm{Mie})}$ is calculated from Mie theory. Fig. 1a is the plot of error when considering electric dipole $\left(a_{1}\right)$ only, magnetic dipole $\left(b_{1}\right)$ only, and electric quadrupole $\left(a_{2}\right)$ only. It can be seen that the electric dipoles are the main contributors to the backscatter; considering only the magnetic dipole or electric quadrupole will give large error. When particle size increases to the resonance region where the magnetic dipole cannot be neglected, the error will be very large. It originated from the neglect of the magnetic dipole, as confirmed in Fig. 1b where the summation effect of electric and magnetic dipoles is considered. After considering the magnetic dipole contribution, the error is dramatically decreased in the resonance region, and the electric quadrupoles have negligible effect on the backscatter.

Fig. $1 \mathrm{c}$ is the fractional error of the DDA results relative to the Mie results. It shows a similar trend to Fig. 1a and Fig. 1b, and the maximum error around the resonance region in the DDA code. Considering three plots in Fig. 1, it was found that before the resonance point, the error is close to zero, and after the resonance point, the error tends to be negative, which implies that the calculated backscatter without using higher orders of multipoles tends to be less than true values after the resonance point in the case of spherical particles. The error from the DDA code around the resonance area is less than that from Mie theory using the electric dipole only, and the reason for this is still under investigation.

Three different ice particle shapes are now considered: sphere, cube and a hexagon column as shown in Fig. 2, and they are assumed to be randomly oriented in space. After considering the fractional error magnitude and the computer capacity, the backscatter values may be assumed to be true for them when dipole numbers are 47833 , 32768 and 31050, respectively (Okamoto et al., 1995). Fig. 3 is the plot of backscatter error from DDA for three particle shapes and wavelength of $\lambda=3.2 \mathrm{~mm}$. The resonance region of spherical particle backscatter error is around $r=730 \mu \mathrm{m}$. For the hexagon, 

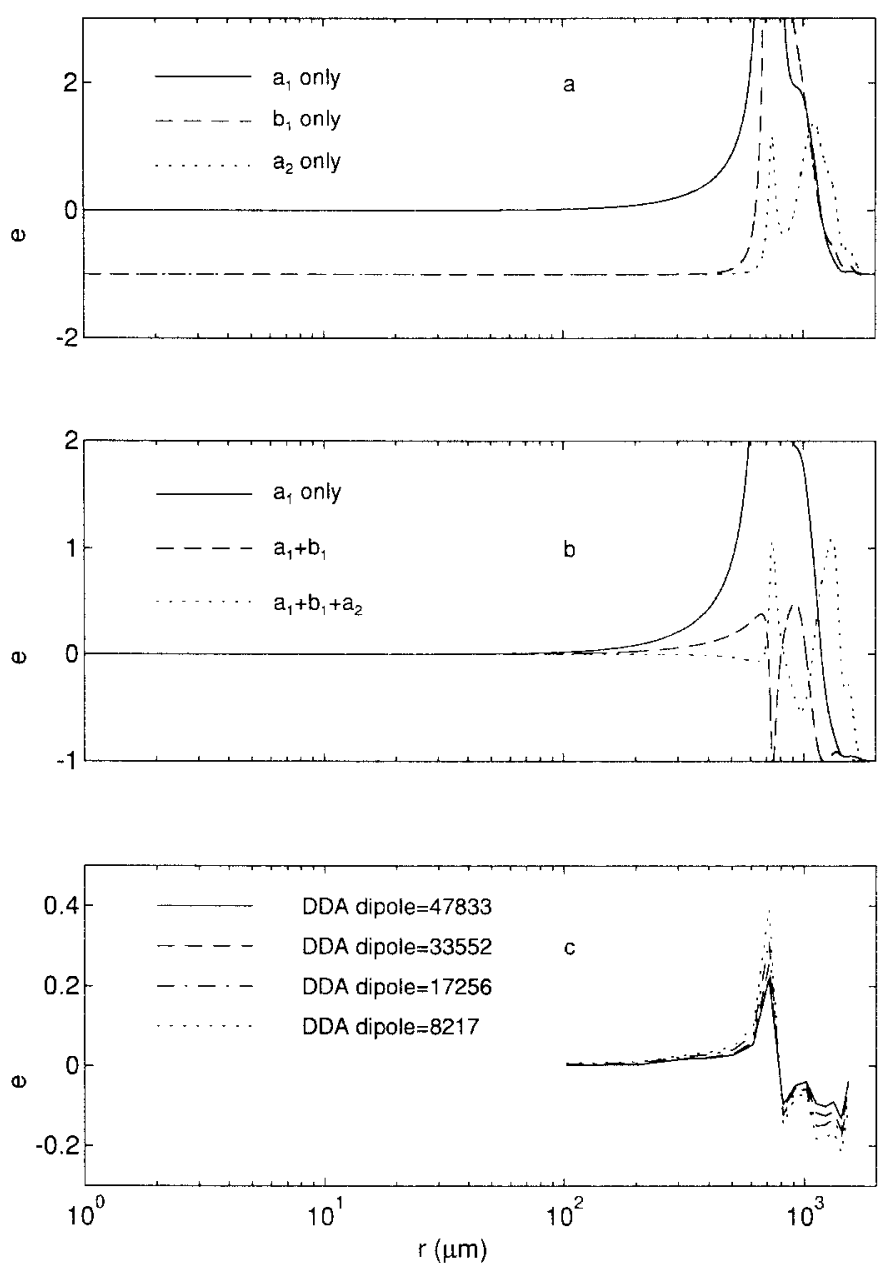

Fig. 1. Spherical ice particle backscatter fractional error variations with particle radius at $\lambda=3.2 \mathrm{~mm}$. The true value $i_{\text {b(Mie) }}$ is calculated from Mie theory. (a) The plot of error when considering electric dipole $\left(a_{1}\right)$ only, magnetic dipole $\left(b_{1}\right)$ only, and electric quadrupole $\left(a_{2}\right)$ only; (b) shows the summation effect of electric dipole, magnetic dipole, and electric quadrupole; $(c)$ is the results from the DDA code.

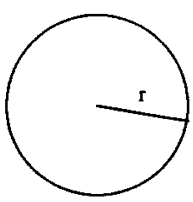

sphere

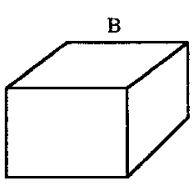

cube

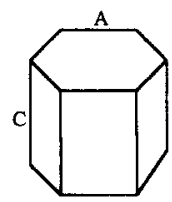

column

Fig. 2. Geometrical diagram of three ice particle shapes considered in our calculation: sphere, cube and hexagon column. 

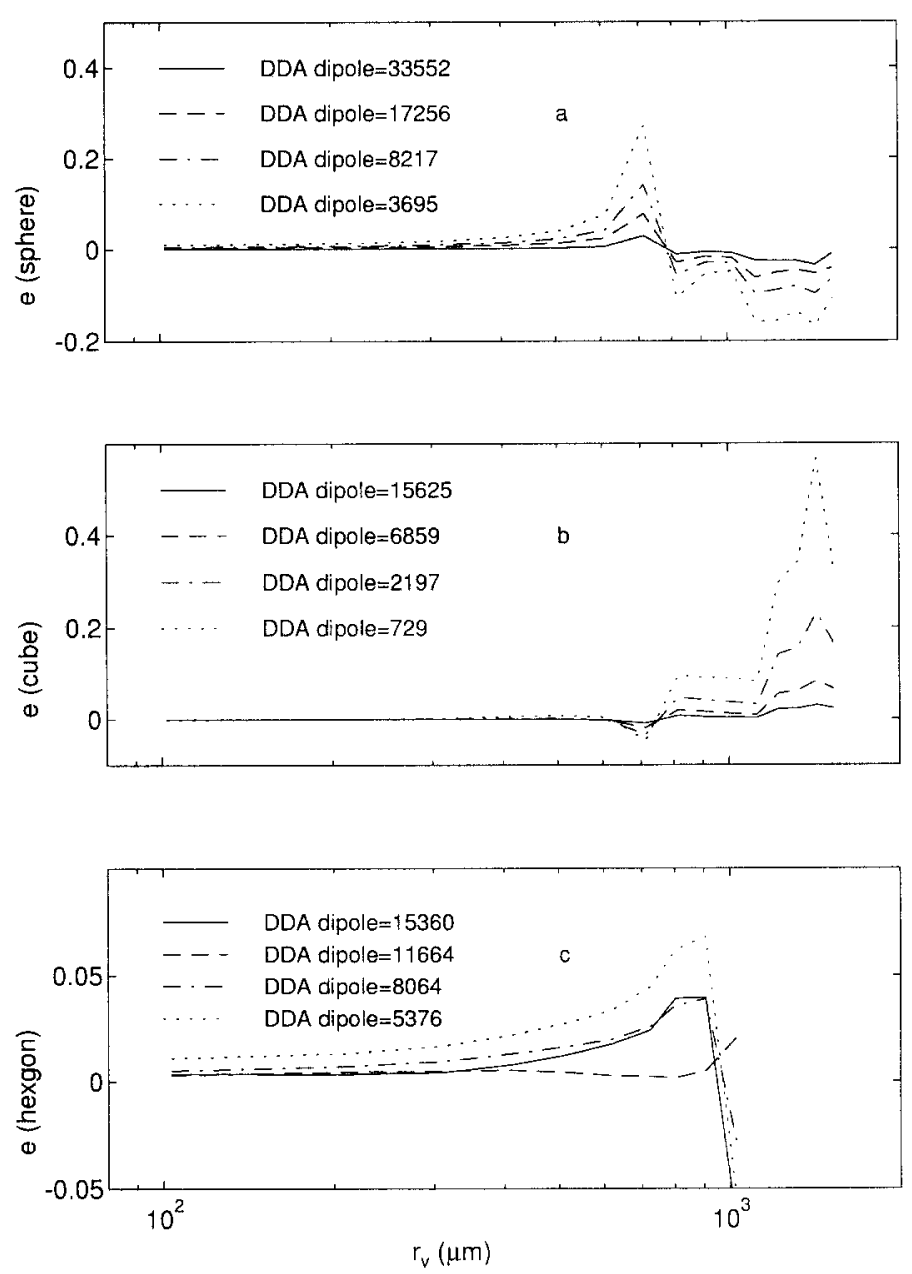

Fig. 3. Ice particle backscatter fractional error variation with volume-equivalent spherical particle radius for three ice particle shapes randomly oriented in space. The wavelength used in this calculation is $\lambda=3.2 \mathrm{~mm}$. (a) For a sphere; (b) for a cube; and (c) for a hexagon with aspect ratio of 3 .

the aspect ratio (length over width $=\mathrm{C} / 2 \mathrm{~A}$ ) is assumed to be 3.0. When the resonance occurs, the volume equivalent particle radius $r_{v}$, and the corresponding particle dimensions are listed in Table 2.

From Fig. 3, it is clear that even for a particle surface which is represented precisely by the cubic dipole array, such as for a cube, a large error still occurs around the resonance point (see Fig. 3b). For a hexagon, the cubic dipole array cannot represent the particle surface accurately, and there is a wider resonance area than those resulting from spherical and cubic particles, but the largest error is also around the resonance region due to the neglect of magnetic dipole contribution to the backscatter in DDA model. The resonance area shifts with the particle shapes. It is noted that the results for the hexagon 
Table 2

Resonance point position for different particle shapes

\begin{tabular}{llll}
\hline Resonance point position $(\mu \mathrm{m})$ & & \\
\hline Particle dimension $(\mu \mathrm{m})$ & Sphere & Cube & Hexagon \\
\hline$r_{\mathrm{v}}$ & 734 & 1200 & 1050 \\
B & & 1934 & \\
A & & & 678 \\
C & & & 4065 \\
\hline
\end{tabular}
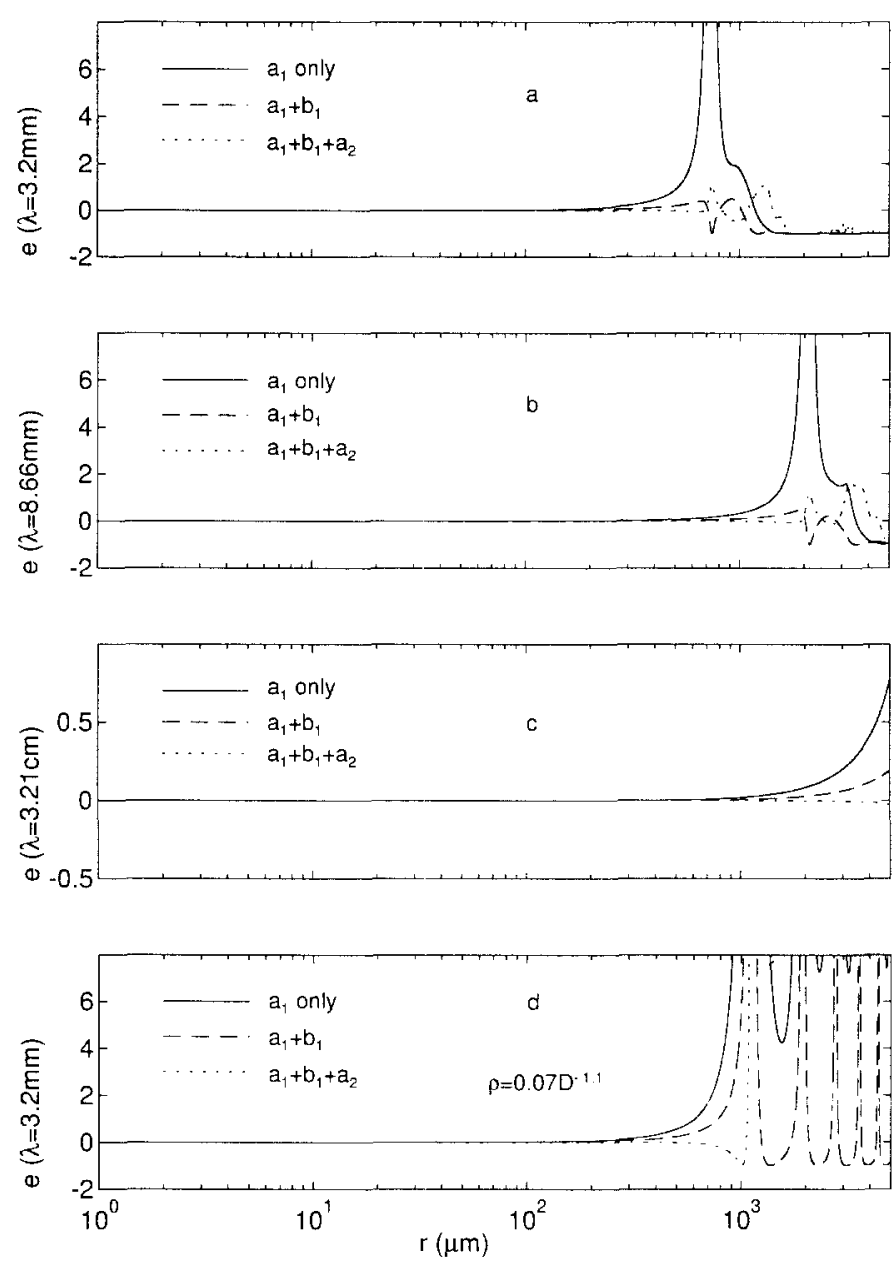

Fig. 4. Ice particle backscatter fractional error variations with spherical particle radius when considering contributions from electric dipole $\left(a_{1}\right)$, magnetic dipole $\left(b_{1}\right)$ and electric quadrupole $\left(a_{2}\right)$, respectively. Wavelengths used in the calculations are (a) $\lambda=3.2 \mathrm{~mm}$, (b) $8.66 \mathrm{~mm}$, and (c) $3.21 \mathrm{~cm}$, respectively. (d) The error variation with the particle radius after considering the ice particle density variation with the particle diameter at $\lambda=3.2 \mathrm{~mm}$. 
(shown In Fig. 3c) does not converge to zero in the resonance region, which is also shown in the results of Okamoto et al. (1995). This may because there are not enough dipoles used in the DDA code due to computer capacity limit. For the hexagon considered in this paper, the resonance occurs around the particle maximum size dimension of $4065 \mu \mathrm{m}$, so the resonance area may have little effect on the bulk particle backscatter considering the low number density of large particles in cirrus cloud.

In order to investigate the resonance position dependence on radar wavelength, three microwave radar wavelengths are used here and they are $3.2 \mathrm{~mm}(94 \mathrm{GHz}), 8.66 \mathrm{~mm}$ ( $K_{\mathrm{a}}$ band) and $3.21 \mathrm{~cm}$ (X-band), respectively. Backscatter errors calculated from Mie theory using spherical particles are plotted in Fig. 4 for particle radius from 1 to 5000 $\mu \mathrm{m}$. It can be seen that the resonance area occurs at larger particle size when the wavelength increased. The reason for this is that the scattering result of Mie theory is determined by both the particle size parameter and the refractive index, and the refractive index does not change very much for these three wavelengths, so the resonance position is mainly determined by the particle size parameter here. For $\lambda=3.2$ mm (Fig. 4a), $8.66 \mathrm{~mm}$ (Fig. 4b) and $3.21 \mathrm{~cm}$ (Fig. 4c), the resonance occurs when particle radii are 734, 2076 and $7693 \mu \mathrm{m}$, and the corresponding particle size parameters are $1.44,1.506$ and 1.505 , respectively. This may be a way to determine which wavelength should be used for different particle size ranges, in order to avoid large backscatter error from the DDA model.

Ice particle density variation with the particle size will affect the particle backscatter

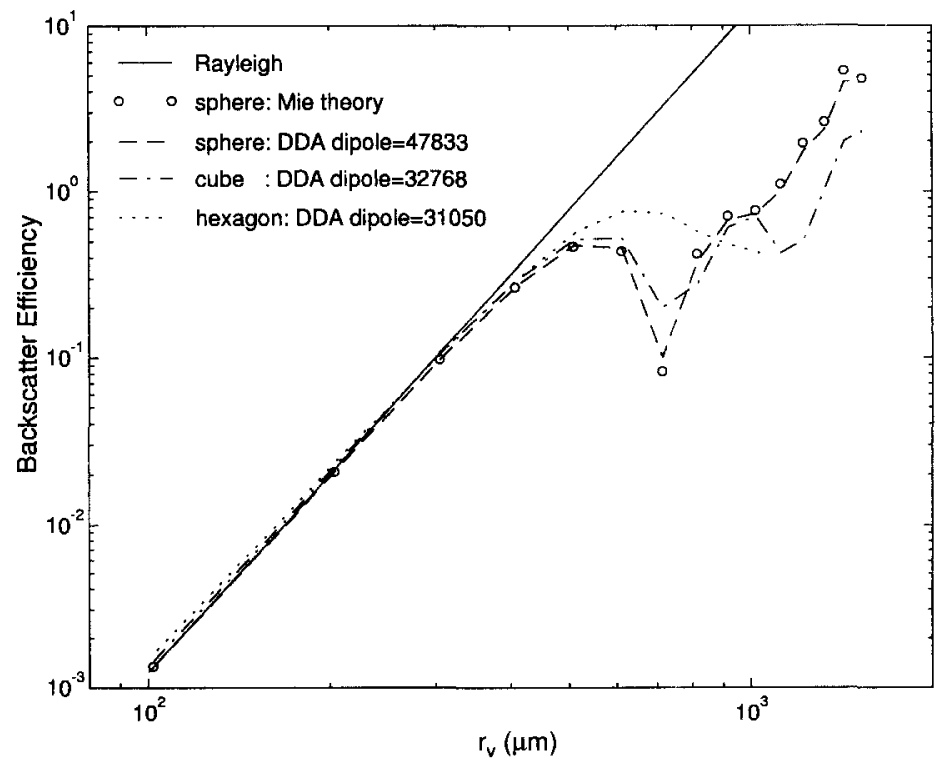

Fig. 5. Backscatter efficiency comparison for different ice particle shapes. Solid line: from Rayleigh formula; circles: from Mie theory for spherical particles; dashed line: from the DDA code for spherical particles; dash-dotted line: from the DDA code for cubes randomly oriented in space; and dotted line: for hexagons with the aspect ratio of 3 and randomly oriented in space. 
(Liu and Illingworth, 1997). The cirrus ice particle density can be written as (Brown and Francis, 1995)

$$
\rho=0.07 D^{-1.1}
$$

where $D$ is particle diameter in millimetres and $\rho$ in $\mathrm{g} \mathrm{cm}^{-3}$. Based on this density distribution, the corresponding ice particle refractive index can be calculated from Maxwell-Garnett (1904) formula, and the ice particle refractive index will decreases with the increase of the particle diameter. After considering the particle density effect on the refractive index, Fig. $4 \mathrm{a}$ is re-calculated and re-plotted in Fig. 4d. It can be seen that the resonance occurs at a similar position as in Fig. 4a, and there is a broad area of large errors after the resonance position. This implies that the lower ice particle refractive index will result in larger backscatter error after the resonance point.

The particle backscatter efficiency (defined as the ratio between the particle backscatter coefficient and the value of $\pi r_{v}^{2}$ ) is plotted in Fig. 5 for three particle shapes at $\lambda=3.2 \mathrm{~mm}$. It can be seen that the backscatter from all three particles are very close for $r_{\mathrm{v}}<500 \mu \mathrm{m}$. The Rayleigh backscatter closely approximates all particle shapes for $r_{\mathrm{v}}<300 \mu \mathrm{m}$. When $r_{\mathrm{v}}>500 \mu \mathrm{m}$, different ice particle shapes will have different backscatter efficiencies.

\section{Summary}

In this paper, DDA code and Mie theory are used to calculate the spherical particle backscatter at wavelength of $3.2 \mathrm{~mm}$, and it was found that the neglect of the magnetic dipole contribution to the particle backscatter will produce errors in backscatter in the resonance region, which occurs around particle size parameter of 1.5. By comparing results from both codes, it is clear that the error from the neglect of the magnetic dipole term is much larger than that from the inaccurate representation of the particle surface shape, and this is also confirmed by the error analysis of cubic and hexagon particle backscatter. No matter how accurate the particle surface shape can be represented, a large error will occur due to the neglect of the magnetic dipole contribution to the backscatter. The error around the resonance region from the DDA code is much lower than that from Mie theory when only considering the electric dipole contribution, the reason for this is still under investigation. The error is expected to be large beyond the resonance region for lower density ice particles.

The significance of the resonance region effect on the bulk particle backscatter will depend upon the region position. From our calculations, for $\lambda=3.21 \mathrm{~cm}$, the resonance region will have little effect on the backscatter due to few particles within that region; for $\lambda=3.2$ and $8.66 \mathrm{~mm}$, this effect may be small considering that the ice particle density decreases with the particle size in cirrus cloud, so the small particles dominate the backscatter, the resonance area effect on the total particle backscatter may be neglected.

The resonance position depends upon the particle aspect ratio of different particle shapes, and this should be further studied, in order to apply DDA code to realistic ice 
particle shapes. The particle density variation (Brown and Francis, 1995) effect on the resonance position should also be further investigated in future studies.

\section{Acknowledgements}

We thank Professor Bruce T. Draine who provided us the latest DDA code. We are also grateful to Mark Blackman for his various assistance in our computations. This work has been supported by NERC grant GR3/8765, ESTEC 10568/93 and CEC EV5V CT94-0463.

\section{References}

Atlas, D., Matrosov, S.Y., Heymsfield, A.J., Chou, M.D., Wolff, D.B., 1995. Radar and radiation properties of ice clouds. J. Appl. Meteor. 34, 2329-2345.

Bohren, C.F. and Huffman, D.R., 1983. Absorption and Scattering of Light by Small Particles. Wiley, New York.

Brown, P.R.A., Francis, P.N., 1995. Improved measurement of the ice water content in cirrus using a total water evaporator. J. Atmos. Oceanic Technol. 12, 410-414.

Brown, P.R.A., Illingworth, A.J., Heymsfield, A.J., McFarquhar, G.M., Browning, K.A., Gosset, M., 1995. The role of spaceborne millimetre-wave radar in global monitoring of ice cloud. J. Appl. Meteor. 34, 2346-2366.

Draine, B.T., 1988. The discrete dipole approximation and its application to interstellar graphite grains. Astrophys. J. 333, 848-872.

Draine, B.T., Flatau, P.J., 1994. Discrete-dipole approximation and its application for scattering calculations. J. Opt. Soc. Am. A 11, 1491-1499.

Dungey, C.E., Bohren, C.F., 1993. Backscattering by nonspherical hydrometeors as calculated by the coupled-dipole method: An application in radar meteorology. J. Atmos. Oceanic Technol. 10, 526-532.

Evans, K.F., Stephens, G.L., 1995. Microwave radiative transfer through clouds composed of realistically shaped ice crystals. Part I: Single scattering properties. J. Atmos. Sci. 52, 2041-2057.

Flatau, P.J., Fuller, K.A., Mackowski, D.W., 1993. Scattering by two spheres in contact: Comparisons between discrete-dipole approximation and modal analysis. Appl. Opt. 32, 3302-3305.

Goedecke, G.H., O'Brien, S.G., 1988. Scattering by irregular inhomogeneous particles via the digitized Green's function algorithm. Appl. Opt. 27, 2439-2444.

Heymsfield, A.J., Platt, C.M.R., 1984. A parameterization of the particle size spectrum of ice cloud in terms of ambient temperature and the ice water content. J. Atmos. Sci. 41, 846-855.

Intrieri, J.M., Stephens, G.L., Eberhard, W.L., Uttal, T., 1993. A method for determining cirrus cloud particle size using lidar and radar backscatter technique. J. Appl. Meteor. 32, 1074-1082.

Liebe, H.J., Manabe, T., Hufford, G.A., 1989. Millimeter-wave attenuation and delay rates due to fog/cloud conditions. IEEE Trans. Antennas and Propagation 37, 1617-1623.

Liou, K.N., 1986. Influence of cirrus clouds on weather and climate processes: A global perspective. Mon. Wea. Rev. 114, 1167-1199.

Liu, C.L. and Illingworth, A.J., 1997. Theoretical studies of ice particle density effect on radar/lidar backscattering of cirrus (in preparation).

Liu, C.L., Illingworth, A.J. and Brown, P.R.A., 1997. Ice water content estimation from cloud temperature and radar reflectivity (in preparation).

Magono, C. and Lee, C.W., 1966. J. Faculty Sci. Hokkaido University, Japan, Series VII (Geophysics), 2 (4).

Maxwell-Garnett, J.C., 1904. Colours in metal glasses and in metallic films. Philos. Trans. R. Soc. A 203, $385-420$. 
Mie, G., 1908. Beigrade zur optik trüber medien, speziell kolloidaler metallósungen. Ann. Physik. 25 (4), $377-445$.

Okamoto, H., Macke, A., Quante, M., Raschke, E., 1995. Modelling of backscattering by non-spherical ice particles for the interpretation of cloud radar signals at $94 \mathrm{GHz}$. An error analysis. Contribution to atmospheric physics $68,319-334$.

Purcell, E.M., Pennypacker, C.R., 1973. Scattering and absorption of light by nonspherical dielectric grains. Astrophys. J. 186, 705-714.

Ray, P.S., 1972. Broadband complex refractive indices of ice and water. Appl. Opt. 11, 1836-1844.

Schneider, T.L., Stephens, G.L., 1995. Theoretical aspects of modelling backscattering by cirrus ice particles at millimetre wavelengths. J. Atmos. Sci. 52, 4367-4385.

Singham, S.B., Salzman, G.C., 1986. The coupled dipole approximation. J. Chem. Phys. 84 (5), 2658-2667.

Singham, S.B., Bohren, C.F., 1987. Light scattering by an arbitrary particle: A physical reformulation of the coupled dipole method. Opt. Lett. 12, 10-12.

Stephens, G.L., Tsay, S., Stackhouse, P.W. Jr., Flatau, P., 1990. The relevance of the microphysical and radiative properties of cirrus clouds to climate and climatic feedback. J. Atmos. Sci. 47, 1742-1753. 